BMJ Open

Respiratory

Research

\title{
Effect of a pulmonary rehabilitation programme of 8 weeks compared to 12 weeks duration on exercise capacity in people with chronic obstructive pulmonary disease (PuRe Duration): protocol for a randomised controlled trial
}

Joshua Bishop, ${ }^{1,2}$ Lissa Spencer, ${ }^{2,3}$ Jennifer Alison ${ }^{2,3}$

To cite: Bishop J, Spencer L, Alison J. Effect of a pulmonary rehabilitation programme of 8 weeks compared to 12 weeks duration on exercise capacity in people with chronic obstructive pulmonary disease (PuRe Duration): protocol for a randomised controlled trial. BMJ Open Resp Res 2020;7:e000687. doi:10.1136/ bmjresp-2020-000687

Received 22 June 2020 Revised 11 August 2020 Accepted 18 August 2020
Check for updates

(c) Author(s) (or their employer(s)) 2020. Re-use permitted under CC BY-NC. No commercial re-use. See rights and permissions. Published by BMJ.

${ }^{1}$ Physiotherapy, Balmain Hospital, Balmain, New South Wales, Australia

${ }^{2}$ Physiotherapy, The University of Sydney Faculty of Medicine and Health, Sydney, New South Wales, Australia

${ }^{3}$ Physiotherapy, Royal Prince Alfred Hospital, Camperdown, New South Wales, Australia

Correspondence to Joshua Bishop; joshua.bishop@health.nsw. gov.au

\section{ABSTRACT}

Introduction Pulmonary rehabilitation (PR) is a key component in the management of chronic obstructive pulmonary disease (COPD). There is no strong evidence on the optimal duration of PR programmes. The aim of this study is to determine whether an 8-week PR programme is equivalent to a 12-week PR programme in people with COPD.

Methods and analysis This study will be a prospective multisite, randomised controlled, equivalence trial with assessors blinded to group allocation and intentionto-treat analysis. 72 participants with COPD will be recruited and randomised to either a supervised, twice weekly for 8 weeks or a 12-week PR programme of exercise training and education. Primary outcome: endurance shuttle walk test. Secondary outcomes: will include St George's Respiratory Questionnaire, 6-min walk distance, COPD assessment test, Hospital Anxiety and Depression Scale, physical activity monitoring and hospital admissions at 6 months and 12 months. Repeated measures analysis of variance will be used to analyse differences between the groups for all outcomes.

Ethics and dissemination Ethics approval was gained from all participating sites. Results of the trial will be submitted for publication in a peer-reviewed journal. Trial registration number ACTRN12616001586404.

\section{INTRODUCTION}

Chronic respiratory diseases are among the leading causes of morbidity and mortality worldwide. Chronic obstructive pulmonary disease (COPD) is ranked eighth in causes of disease burden around the world, ${ }^{1}$ and is one of the leading causes of death by a non-communicable diseases. ${ }^{2}$ Currently, there is no cure for COPD, so management should involve both pharmacological and non-pharmacological interventions which can prevent decline of physical function and improve health-related quality of life (HRQoL). ${ }^{3}$

Pulmonary rehabilitation (PR) is the key, non-pharmacological component in the management of COPD and has been shown to improve breathlessness, HRQoL and exercise capacity and reduce hospital admissions and length of stay in people with COPD. ${ }^{45}$ The duration of $\mathrm{PR}$ programmes reported in randomised controlled trials (RCTs) varies greatly, from 4 weeks to 18 months, with the majority of these being between 6 and 12 weeks in duration. ${ }^{6}$ PR programmes of 8 weeks duration are recommended in guidelines and statements; ${ }^{6-9}$ however, there is no strong evidence on the optimal duration of PR programmes. The most recent Cochrane review on PR for $\mathrm{COPD}^{4}$ stated that the optimal duration of $\mathrm{PR}$ programmes is unclear as the available evidence was insufficient to determine this.

There have been several trials that have studied the effects of the duration of PR programmes on exercise capacity and HRQoL; however, a systematic review of these RCTs ${ }^{10}$ was not able to perform a metaanalysis due to heterogeneity in programme durations and outcome measures, therefore no strong recommendations on programme duration are available. Of the five studies included in the systematic review, two compared a 4-week supervised exercise programme to a 7-week programme in people with COPD, ${ }^{11} 12$ one compared 8 weeks to 20 weeks $^{13}$ and two compared 12 weeks to 72 weeks (18 months). ${ }^{14}{ }^{15}$ While 


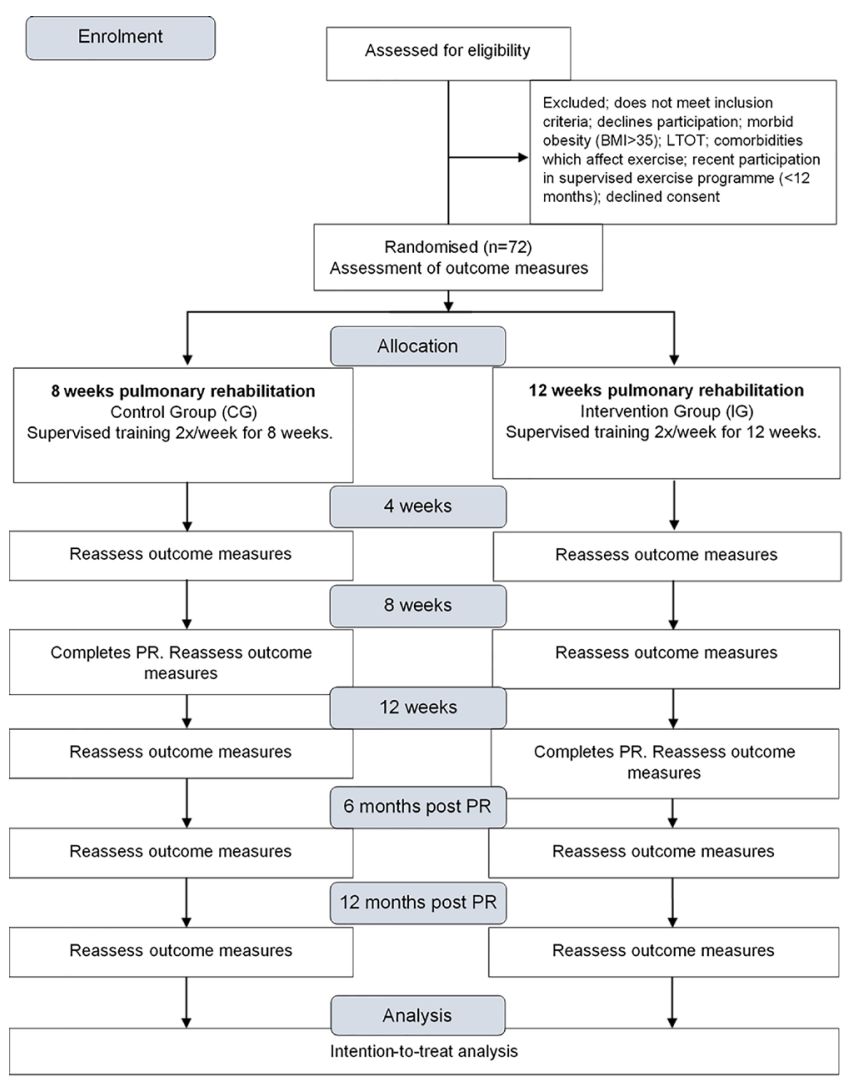

Figure 1 CONSORT flowchart. 6 months assessment is 6 months from the end of the respective programme and the 12 months assessment is 12 months from the end of the respective programme. BMI: body mass index; CONSORT, Consolidated Standards of Reporting Trials; LTOT: longterm oxygen therapy; PR: pulmonary rehabilitation; 2x/ week: two times per week.

the quality of most of these studies was low, the overall conclusion was that comparatively longer programmes showed slightly better improvements in HRQoL than shorter programmes, while results for differences in exercise capacity were equivocal. Longer PR programmes are more expensive to deliver, and current evidence cannot determine whether longer programmes provide significantly greater benefits for people with COPD in terms of both exercise capacity and HRQoL.

PR statements and guidelines highlighted that more research is needed to determine the optimal duration of PR programmes to ensure the best outcomes for people with COPD. ${ }^{79}$ As the most common recommendation is for programmes of 8 weeks duration, the aim of this study is to determine whether an 8-week PR programme is equivalent to a 12-week programme in improving exercise capacity (primary outcome) in people with COPD.

The primary hypotheses are: (1) that endurance exercise capacity at the end of the 8-week PR programme will be equivalent to that at the end of the 12-week programme; (2) that endurance exercise capacity measured at 12 weeks will be equivalent for both the 8-week PR programme and the 12-week programme; and
(3) that endurance exercise capacity will be equivalent between groups at the 6 months and 12 months follow-up.

\section{METHODS AND ANALYSIS \\ Design}

This study will be a prospective, multisite, randomised controlled, equivalence trial with assessors blinded to group allocation and intention to treat analysis.

\section{Participants}

People will be eligible to participate if they have: a diagnosis of COPD (post bronchodilator forced expiratory volume in $1 \mathrm{~s}\left(\mathrm{FEV}_{1}\right)$ to forced vital capacity (FVC) ratio of $<0.7 ; \mathrm{FEV}_{1}<80 \%$ predicted. ${ }^{3}$ People with COPD will be excluded if they have: a body mass index of greater than $35 \mathrm{~kg} / \mathrm{m}^{2}$; been prescribed long-term oxygen therapy (LTOT); significant comorbidities; musculoskeletal, cardiovascular or neurological conditions which would adversely affect their ability to participate in the exercise assessments or exercise programmes; or participated in a supervised exercise programme within the last 12 months.

The study protocol will follow the recommendations of the Consolidated Standards of Reporting Trials (CONSORT) guidelines for an equivalence trial ${ }^{16}$ and participant flow is outlined in figure 1. Written and verbal information will be provided to participants, and written consent will be obtained from all participants by the physiotherapists running the PR programmes at each site prior to enrolment. The study has been approved by the Human Research and Ethics Committee of Sydney Local Health District. The trial is registered with the Australian and New Zealand Clinical Trials Registry: ACTRN12616001586404. Ethics approval will be sought for any changes to the study protocol and such changes will be updated in the trial registry.

\section{Recruitment}

Participants will be recruited through referrals to the PR programme at three sites within a large health district in Sydney, Australia. Participants will be screened for eligibility prior to enrolment in the trial.

\section{Randomisation and blinding}

Following assessment of baseline measures, sequence generation by a therapist external to the study, will be determined using a computer-generated randomisation programme with a minimisation algorithm, stratifying for site, sex, $\mathrm{FEV}_{1}<50 \%$ predicted, 6 -min walk distance $<350 \mathrm{~m}$, oxygen saturation $\left(\mathrm{Sp}_{2}\right)$ during 6 -min walk test $(6 \mathrm{MWT})<90 \%$ and an exacerbation of COPD occurring during the previous 4 weeks. The physiotherapists at each PR site will be contacted and informed of the group allocation. Assessors and statistician will be blinded to group allocation. 


\section{Intervention and control groups}

Both the 8-week PR group (control group (CG)) and the 12-week PR group (intervention group (IG)) will participate in the same twice weekly, supervised PR programme at all sites. The PR programme will consist of supervised exercise training, education and support for selfmanagement. ${ }^{7}$ The IG will receive an additional 4 weeks of twice weekly, supervised exercise training.

\section{Exercise training}

The PR programmes will be held in hospital outpatient settings, and supervised by physiotherapists experienced in the management of people with COPD and in delivering PR programmes. The duration of each training session will be up to 1.5 hours, including rest.

Exercise training will consist of overground or treadmill walking, lower limb stationary cycling, lower and upper limb strengthening exercises which will be prescribed individually and relative to each participant's initial assessment.

Initial exercise intensities will be standardised for each modality:

- Walking (overground or treadmill) will begin at $80 \%$ of each participant's average walk speed determined by the $6 \mathrm{MWT}^{17}$ and progressed based on symptoms of dyspnoea and perceived exertion, with the aim that the participants will score a dyspnoea or rate of perceived exertion (RPE) score of at least 3 to 4 ('moderate' to 'somewhat severe') on the modified 0 to 10 category-ratio scale. ${ }^{18}$ Initial walking duration will be $15 \mathrm{~min}$ and will be progressed to $30 \mathrm{~min}$ between weeks 2 and 4 .

- Lower limb cycling intensity will commence at $60 \%$ of each participant's estimated peak work rate using an equation based on the results of the $6 \mathrm{MWT}^{19}$ and progressed to $70 \%$ peak work rate based on symptoms of dyspnoea and RPE, with the aim of participants scoring a dyspnoea or RPE score of at least 3 to 4 on the modified 0 to 10 category-ratio scale. ${ }^{1820}$

- Arm ergometry will be performed for $10 \mathrm{~min}$ at an intensity of 3 to 4 on the RPE 0 to 10 scale.

- Lower limb strengthening exercises using body weight (squats, sit-to-stands and step-ups) and arm exercises using free weights will commence in week 2 at a load that can be achieved for 8 to 10 repetitions before muscle exhaustion. Fixed weight strengthening exercises for the lower limbs (leg extension and leg press) and upper limbs (latissimus dorsi pull downs and chest press/fly) will commence in week 3 at a load that can be achieved for 8 to 10 repetitions before local muscle exhaustion. By week 4, all strengthening exercise (body weight, free weight and fixed weight exercises) will be completed three times (ie, three sets). Resistances will be increased once participants can achieve three sets of 10 repetitions in two consecutive training sessions.
All participants will receive individualised (one-on-one) education, as applicable, on the following topics: how the lungs work, what is COPD, medication use, managing breathlessness, importance of exercise training, smoking cessation (if current smokers), sputum clearance (if appropriate), symptom monitoring and exacerbation management. Participants will be encouraged to walk on the days they do not attend the formal programme. At completion of the programme ( 8 weeks and 12 weeks) participants will be given a booklet with information on continuing a walking programme at least 3 days a week and simple body weight exercises (as performed during the programme). The booklet will encourage monitoring their symptoms, include a COPD action plan and advise them to seek early review with their doctors if they experience an exacerbation.

Attendance will be supervised and monitored by the physiotherapists and participants will be encouraged to attend each allocated session.

\section{Primary outcome measures \\ Endurance exercise capacity}

The primary outcome will be endurance exercise capacity measured by the endurance shuttle walk test (ESWT). ${ }^{21}$ The ESWT is an externally paced walking test. The walking speed of the ESWT will be determined by a calculation taken from the participant's walking speed during the $6 \mathrm{MWT} .{ }^{22}$ Participants will be asked to walk back and forth along a 10-m course until they are no longer able to keep up with the pre-determined pace. Dyspnoea and RPE will be measured pre-test and end-test, and heart rate (HR) and $\mathrm{SpO}_{2}$ will be recorded at each minute during the test using a pulse oximeter (Onyx Vantage 9590, Nonin Medical Inc, Plymouth, Minnesota, USA). At the end of the test, participants will be asked the reason for stopping and this will be recorded. The ESWT will be performed twice at baseline and once at every other assessment point. At baseline, participants will rest for at least $30 \mathrm{~min}$ between walking tests. The ESWT has a maximum time of $20 \mathrm{~min}^{21}$ At baseline, if a participant reaches $10 \mathrm{~min}$ comfortably, the test will be ceased, and then after a 30-min rest, the test will be re-started at the next higher speed with the aim of the participant achieving an ESWT time of between 5 and $10 \mathrm{~min}$. This would enable an improvement in ESWT (time) to be recorded at the end of PR without the participant reaching the ESWT ceiling of $20 \mathrm{~min}$. The ESWT speed used during the baseline assessment will be the same speed used at each subsequent assessment time point.

\section{Secondary outcomes measures \\ Health-related quality of life}

HRQoL will be measured using the St. George's Respiratory Questionnaire (SGRQ). ${ }^{23}$ The SGRQ is a selfadministered questionnaire designed to measure selfperceived impairments in health and quality of life in populations who have airways disease. It measures three 
domains (symptoms, activity limitations and impact of disease) and gives a total score between 0 to 100 , where 0 indicates good health and 100 very poor health.

\section{6-minute walk distance}

Functional exercise capacity will be measured using the 6MWT. ${ }^{1724}$ This test measures the distance a participant can walk in $6 \mathrm{~min}$ following a standardised protocol which includes standard instructions, encouragement, the type of track used and the number of tests performed. Participants will be asked to walk as far as they can in $6 \mathrm{~min}$ being notified of how much time is left each minute. $\mathrm{HR}$ and $\mathrm{SpO}_{2}$ will be measured at rest and every minute during the test using a pulse oximeter (Onyx Vantage 9590, Nonin Medical Inc, Plymouth, Minnesota, USA). At the end of the test, participants will be asked if there was anything that limited the walk distance. At baseline, participants will perform two 6MWTs with the greater distance being recorded as the baseline measure. The distance will also be used in an equation to determine the participant's starting level for the ESWT. ${ }^{22}$ At baseline, participants will rest for at least $30 \mathrm{~min}$ between each walking test. The order of exercise tests at baseline will be two 6MWTs followed by two ESWTs.

\section{COPD assessment test}

Participants' symptoms will be recorded using the COPD assessment test (CAT). ${ }^{25}$ This questionnaire has eight items which include questions about: cough, sputum, chest tightness, breathlessness on hills/stairs, activity limitation at home, sleep and fatigue. A higher score indicates greater impact of COPD on the participant's life. ${ }^{26}$

\section{Anxiety and depression}

Anxiety and depression will be measured using the Hospital Anxiety and Depression Scale. ${ }^{27}$ This scale has been shown to be a valid and repeatable measure of anxiety and depression in those attending outpatient clinics. ${ }^{27}$ This scale uses 14 statements (seven related to anxiety, seven to depression) and scores each statement from 0 to 3 , for a maximum score of 21 in each domain. Higher scores indicate a higher likelihood of depression or anxiety, with a cut-off score (in each domain) of $\geq 8$ indicating possible anxiety or depression. ${ }^{28}$

\section{Physical activity}

Physical activity will be measured using two devices worn for 7 days. The devices will be:

1. The ActiGraph GT3X (ActiGraph LLC, Pensacola, Florida, USA) will be used to measure energy expenditure during physical activity and has been validated for use in people with COPD. ${ }^{29}$ This device will be worn over the anterior superior iliac crest and held in place by an elastic belt around the waist. This device is removed during sleep and for water-based activities such as showering/bathing.
2. The activPAL (PAL Technologies Ltd, Glasgow, UK) will be used to count numbers of steps taken and time spent in sitting/lying, standing and walking activities. The activPAL has been validated for the measurement of free living activities in the healthy population ${ }^{30}$ and in COPD. ${ }^{31}$ The activPAL will be in a waterproof cover and worn on the thigh and will be held in place with a medical adhesive covering. This allows the device to be worn for 24 hours a day during any activity, including water-based activities such as showering/bathing.

Data from both devices will be included in analysis if worn for at least 4 days. Data will be collected at baseline, week 7 during the programme, the week following completion of the PR programme, 6-month follow-up and 12-month follow-up.

\section{Hospital admissions and length of stay}

The number of hospital admissions and participant's length of stay will be reviewed at 6 months and 12 months post completion of the PR programmes and compared with a similar period prior to the programme. Hospitalisation data will be collected using the health district's data management system.

\section{Exercise adherence}

Exercise adherence will be measured by counting the number of exercise sessions attended divided by possible number of exercise sessions. Acceptable adherence will be set at $75 \%$ of possible classes attended ${ }^{32}$ that is, 12 sessions for the 8-week PR group and 18 sessions for the 12-week PR group.

\section{Adverse events}

Any adverse events will be immediately reported to the ethics committee. All adverse events will be recorded and reported in the study findings.

\section{Data management and analysis}

To ensure confidentiality, a master code sheet containing participant code and identifying characteristics will be kept by the lead investigator in a locked filing cabinet, separate to all other data. All de-identified study related information will be stored electronically in a secure, password protected Research Electronic Data Capture (REDCap) database. ${ }^{33} 34$ Original paper files with participant code will be stored in numerical order in a locked filing cabinet within an office that will be locked overnight. While study findings will be presented at relevant conferences and published in peer-reviewed journals, only group data, in which individual participants will not be identifiable, will be used.

If a participant declines to perform an exercise test, then every effort will be made to at least collect questionnaire data. Intention-to-treat analysis will be used; therefore, data from participants who deviate from the allocated intervention will be included. 
For all outcome measures, the analysis will be completed using Statistical Package for the Social Sciences (SPSS) software (V.25.0.0.2, IBM Corporation, Armonk, New York, USA). Normally distributed data will be expressed as means and SD. Repeated measures analysis of variance (ANOVA) will be used to analyse differences between the groups for all continuous outcomes. Independent sample t-tests will assess the differences between groups at specific time points. A Mann-Whitney U-test will be used to compare the number of hospital admissions and compare hospital lengths of stay. Baseline data from participants who do not complete the study will be compared with the baseline data from those who complete, to determine whether withdrawals bias the findings.

\section{Sample size calculation}

A total of 72 participants (36 per group) will be recruited to ensure that 58 participants will complete the study, allowing for a $25 \%$ drop out rate that may be anticipated in the long-term study. Fifty-eight participants are required to be $80 \%$ sure that the two-sided CI with a significance level of $5 \%$ will exclude a difference of the mean ESWT time by $186 \mathrm{~s}$ previously reported as a minimum important difference in ESWT following exercise training in COPD. ${ }^{2435}$ This assumes an SD of $239 \mathrm{~s}$ for the ESWT. ${ }^{36}$

\section{Patient and public involvement}

This trial will involve consumer representatives in the development and dissemination of a summary paper, written in lay language for participants and the wider community.

\section{ETHICS AND DISSEIMINATION}

Written and verbal information will be provided to participants, and, prior to enrolment, written consent will be obtained from all participants by the physiotherapists running the PR programme at each site. The study has been approved by the Human Research and Ethics Committee of Sydney Local Health District. The trial is registered with the Australian and New Zealand Clinical Trials Registry: ACTRN12616001586404. Ethics approval will be sought for any changes to the study protocol and such changes will be updated in the trial registry.

A manuscript with the primary results from the study will be submitted for publication in a peer-reviewed journal. Submissions will also be written to present at national and international conferences to further disseminate results.

\section{DISCUSSION}

The primary aim of this study is to determine whether the recommended 8-week PR programme is equivalent to a 12-week programme in improving endurance exercise capacity. We hypothesise that there will be equivalence in endurance exercise capacity between the groups at the completion of their respective PR programmes (8 weeks and 12 weeks).

No RCTs have compared the recommended 8-week PR programme to a longer PR programme. Two previous RCTs have compared a short 4-week PR programme to a 7-week programme. ${ }^{11} 12$ Green et al compared outcomes at the respective completion point of both groups (ie, the outcomes of the 4-week PR programme were compared with the outcomes of the 7-week programme) and reported an improvement in HRQoL in favour of the 7-week programme while no significant between-group difference in exercise capacity was reported. ${ }^{11}$ The other RCT by Sewell et al was performed at the same centre and compared the 4-week and 7-week PR programmes at two time points, that is at completion and at 7 weeks for both groups (in which the 4-week group had followed a 3-week home exercise programme). ${ }^{12}$ This study reported different findings from the first study with no significant differences between groups for HRQoL at the 7-week time point, and a significant difference between groups in the ESWT favouring the 4-week programme at the 7-week time point. These studies suggest that both HRQoL and exercise capacity can improve over a short period of time but due to the differing results, it is not clear whether a longer or shorter duration PR programme would be more beneficial.

There is also some limited evidence for greater improvements in exercise capacity and HRQoL with programmes longer than 8 weeks and 12 weeks when compared with shorter programmes. However the quality of the evidence in these studies was poor ${ }^{13-15}$ and therefore statements and guidelines cannot draw appropriate conclusions on the optimal length of PR programmes for people with COPD.

The time course of improvement in exercise capacity and HRQoL during an 8-week PR programme has been reported in a recent retrospective study. ${ }^{37}$ The study showed that participants demonstrated improvements in the 6MWT at both 4 weeks and 8 weeks compared with baseline which were greater than the minimal clinically important difference of $30 \mathrm{~m}^{24}$ at both time points. These improvements were maintained at the 1-month follow-up and 2-month follow-up for both the moderatefunctioning and high-functioning participants (classified as having higher baseline $6 \mathrm{MWT}^{\mathrm{FEV}} \mathrm{F}_{1} \%$ predicted, greater leg strength and less requirement for LTOT), while the low-functioning participants did not maintain their improvements in the 6MWT at either of these follow-up times. This study demonstrated that while participants in an 8-week PR programme could improve function, those with lower levels of function were unable to maintain these changes and therefore different durations of PR programmes may be needed to suit different patients' disease severity and limitations.

By collecting data regarding exercise capacity, HRQoL, physical activity and hospital admissions, the proposed 
study will provide insight into the optimal duration of PR programmes. If participating in an 8-week PR programme has equivalent outcomes to a 12-week programme in improving exercise capacity and HRQoL, and if maintenance of these changes is also equivalent, then the 8-week programme would be justified. On the contrary, if the 12-week PR programme is shown to have greater improvements in the primary outcomes, then these findings will help to determine if it is necessary to change the recommended length of a PR programme. Since longer programmes are more expensive to deliver and require a longer commitment from participants and health professionals, there needs to be strong evidence of both shortterm and long-term benefits from longer programmes if they are to be implemented. Information regarding hospital admissions and length of stay will only be exploratory, as the study is not powered for this outcome.

This study will fill a significant knowledge gap in the field of PR. No RCTs have directly evaluated the 8-week PR programmes compared with longer PR programmes. Data from this study will provide clinicians and policymakers with important guidance on the most effective duration of PR programmes for people with COPD.

Contributors JB, LS and JA designed the trial protocol. JB procured funding for the study with assistance from LS and JA. JB, LS and JA drafted the manuscript. All authors read and approved the final manuscript.

Funding Sydney Local Health District Allied Health Seeding Grant. Physiotherapy Research Foundation Seeding Grant (Project Grant S17 - 019).

Competing interests None declared.

Patient and public involvement Patients and/or the public were involved in the design, or conduct, or reporting, or dissemination plans of this research. Refer to the Methods section for further details.

Patient consent for publication Not required.

Provenance and peer review Not commissioned; externally peer-reviewed.

Open access This is an open access article distributed in accordance with the Creative Commons Attribution Non Commercial (CC BY-NC 4.0) license, which permits others to distribute, remix, adapt, build upon this work non-commercially, and license their derivative works on different terms, provided the original work is properly cited, appropriate credit is given, any changes made indicated, and the use is non-commercial. See: http://creativecommons.org/licenses/by-nc/4.0/.

\section{REFERENCES}

1 GBD 2015 Disease and Injury Incidence and Prevalence Collaborators. Global, regional, and national incidence, prevalence, and years lived with disability for 310 diseases and injuries, 19902015: a systematic analysis for the global burden of disease study 2015. Lancet 2016;388:1545-602.

2 GBD 2015 Mortality and Causes of Death Collaborators. Global, regional, and national life expectancy, all-cause mortality, and causespecific mortality for 249 causes of death, 1980-2015: a systematic analysis for the global burden of disease study 2015. Lancet 2016;388:1459-544.

3 Vogelmeier CF, Criner GJ, Martinez FJ, et al. Global strategy for the diagnosis, management, and prevention of chronic obstructive lung disease 2017 report: gold executive summary. Eur Respir J 2017;49:1700214.

4 McCarthy B, Casey D, Devane D, et al. Pulmonary rehabilitation for chronic obstructive pulmonary disease. Cochrane Database Syst Rev 2015:CD003793.

5 Puhan MA, Gimeno-Santos E, Cates CJ, et al. Pulmonary rehabilitation following exacerbations of chronic obstructive pulmonary disease. Cochrane Database Syst Rev 2016;12:CD005305.
6 Bolton CE, Bevan-Smith EF, Blakey JD, et al. British thoracic Society guideline on pulmonary rehabilitation in adults. Thorax 2013;68:ii1-30.

7 Spruit MA, Singh SJ, Garvey C, et al. An official American thoracic Society/European respiratory Society statement: key concepts and advances in pulmonary rehabilitation. Am J Respir Crit Care Med 2013;188:e13-64.

8 Marciniuk DD, Brooks D, Butcher S, et al. Optimizing pulmonary rehabilitation in chronic obstructive pulmonary disease--practical issues: a Canadian Thoracic Society Clinical Practice Guideline. Can Respir J 2010;17:159-68.

9 Alison JA, McKeough ZJ, Johnston K, et al. Australian and New Zealand pulmonary rehabilitation guidelines. Respirology 2017:22:800-19.

10 Beauchamp MK, Janaudis-Ferreira T, Goldstein RS, et al. Optimal duration of pulmonary rehabilitation for individuals with chronic obstructive pulmonary disease - a systematic review. Chron Respir Dis 2011;8:129-40.

11 Green RH, Singh SJ, Williams J, et al. A randomised controlled trial of four weeks versus seven weeks of pulmonary rehabilitation in chronic obstructive pulmonary disease. Thorax 2001;56:143-5.

12 Sewell L, Singh SJ, Williams JEA, et al. How long should outpatient pulmonary rehabilitation be? a randomised controlled trial of 4 weeks versus 7 weeks. Thorax 2006;61:767-71.

13 Swerts PM, Kretzers LM, Terpstra-Lindeman E, et al. Exercise reconditioning in the rehabilitation of patients with chronic obstructive pulmonary disease: a short- and long-term analysis. Arch Phys Med Rehabil 1990;71:570-3.

14 Foy CG, Rejeski WJ, Berry MJ, et al. Gender moderates the effects of exercise therapy on health-related quality of life among COPD patients. Chest 2001;119:70-6.

15 Berry MJ, Rejeski WJ, Adair NE, et al. A randomized, controlled trial comparing long-term and short-term exercise in patients with chronic obstructive pulmonary disease. J Cardiopulm Rehabil 2003;23:60-8.

16 Piaggio G, Elbourne DR, Pocock SJ, et al. Reporting of noninferiority and equivalence randomized trials: extension of the CONSORT 2010 statement. JAMA 2012;308:2594-604.

17 ATS Committee on Proficiency Standards for Clinical Pulmonary Function Laboratories. ATS statement: guidelines for the six-minute walk test. Am J Respir Crit Care Med 2002;166:111-7.

18 Borg GA. Psychophysical bases of perceived exertion. Med Sci Sports Exerc 1982;14:377???381-81.

19 Hill K, Jenkins SC, Cecins N, et al. Estimating maximum work rate during incremental cycle ergometry testing from six-minute walk distance in patients with chronic obstructive pulmonary disease. Arch Phys Med Rehabil 2008;89:1782-7.

20 Zainuldin R, Mackey MG, Alison JA. Prescribing cycle exercise intensity using moderate symptom levels in chronic obstructive pulmonary disease. J Cardiopulm Rehabil Prev 2016;36:195-202.

21 Revill SM, Morgan MD, Singh SJ, et al. The endurance shuttle walk: a new field test for the assessment of endurance capacity in chronic obstructive pulmonary disease. Thorax 1999;54:213-22.

22 Wootton SL, Ng C, McKeough ZJ, et al. Estimating endurance shuttle walk test speed using the six-minute walk test in people with chronic obstructive pulmonary disease. Chron Respir Dis 2014;11:89-94.

23 Jones PW, Quirk FH, Baveystock CM. The St George's respiratory questionnaire. Respir Med 1991;85 Suppl B:25-31.

24 Holland AE, Spruit MA, Troosters T, et al. An official European respiratory Society/American thoracic Society technical standard: field walking tests in chronic respiratory disease. Eur Respir $J$ 2014;44:1428-46.

25 Jones PW, Harding G, Berry P, et al. Development and first validation of the COPD assessment test. Eur Respir J 2009;34:648-54.

26 Jones PW, Tabberer M, Chen W-H. Creating scenarios of the impact of COPD and their relationship to COPD assessment test (CAT ${ }^{\mathrm{TM}}$ ) scores. BMC Pulm Med 2011;11:42.

27 Zigmond AS, Snaith RP. The hospital anxiety and depression scale. Acta Psychiatr Scand 1983;67:361-70.

28 Bjelland I, Dahl AA, Haug TT, et al. The validity of the hospital anxiety and depression scale. An updated literature review. J Psychosom Res 2002;52:69-77.

29 Rabinovich RA, Louvaris Z, Raste Y, et al. Validity of physical activity monitors during daily life in patients with COPD. Eur Respir $J$ 2013;42:1205-15.

30 Grant PM, Ryan CG, Tigbe WW, et al. The validation of a novel activity monitor in the measurement of posture and motion during everyday activities. Br J Sports Med 2006;40:992-7.

31 Cindy Ng LW, Jenkins S, Hill K. Accuracy and responsiveness of the stepwatch activity monitor and ActivPAL in patients with 
COPD when walking with and without a rollator. Disabil Rehabil 2012;34:1317-22.

32 Williams MT, Lewis LK, McKeough Z, et al. Reporting of exercise attendance rates for people with chronic obstructive pulmonary disease: a systematic review. Respirology 2014;19:30-7.

33 Harris PA, Taylor R, Minor BL, et al. The REDCap consortium building an international community of software platform partners. $J$ Biomed Inform 2019;95:103208.

34 Harris PA, Taylor R, Thielke R, et al. Research electronic data capture (REDCap)--a metadata-driven methodology and workflow process for providing translational research informatics support. J Biomed Inform 2009;42:377-81.

35 Pepin V, Laviolette L, Brouillard C, et al. Significance of changes in endurance shuttle walking performance. Thorax 2011;66:115-20.

36 Leung RWM, McKeough ZJ, Peters MJ, et al. Short-form Sun-style t'ai chi as an exercise training modality in people with COPD. Eur Respir J 2013;41:1051-7.

37 Lee C-T, Hsieh P-L, Chien M-Y, et al. Trajectories of functional exercise capacity in patients undergoing pulmonary rehabilitation. Int J Chron Obstruct Pulmon Dis 2019;14:863-70. 